\section{Posicionamiento frente a los confinamientos obligatorios y el control social durante la pandemia en Chile: un análisis a partir de perfiles emocionales}

\author{
Positioning in the face of mandatory confinements and social control during the pandemic in \\ Chile: an analysis based on emotional profiles
}

Rodolfo Bachler Silva; Pablo Segovia-Lagos; Constanza Carter

\section{RELIGACIÓN \\ REVISTA DE CIENCIAS SOCIALES Y HUMANIDADES JOURNAL OF SOCIAL SCIENCES AND HUMANITIE
REVISTA DE CENCIAS SOCIAIS E HUMANAS}

INFORMACIÓN

http://doi.org/10.46652/rgn.v6izo.855 ISSN $2477-9083$

Vol. 6 No. 30, 2021. e210855 Quito, Ecuador

Enviado: octubre 16, 2021 Aceptado: diciembre 11, 2021 Publicado: diciembre 16, 2021 Publicación Continua Sección Dossier | Peer Reviewed y validado para esta investigación. Los resultados permitieron identificar seis perfiles emocionales asociados a diferentes condiciones sociodemográficas. En un polo, caracterizado por experiencias de rabia, tristeza y desconfianza frente a las medidas, se encuentra un perfil compuesto por mujeres jóvenes, estudiantes y con bajo nivel de ingresos económicos. En el otro polo, caracterizado por presentar comodidad frente al estado de pandemia y confianza frente a las medidas propuestas, se encuentra un grupo conformado por hombres, con edades entre los 40 y 69 años, con un alto nivel educacional y de elevados ingresos económicos. Entre medio, encontramos cuatro perfiles más, con distintas características emocionales y condiciones sociodemográficas. Se discuten los resultados considerando su aporte para el diseño de políticas públicas para el control de la pandemia.

Palabras clave: COVID-19; emociones; pandemia; políticas públicas; confinamiento

\section{ABSTRACT}

The control of the pandemic generated by COVID-19 rely, to a large extent, on the disposition that people have or not to comply with the measures ordered by the health authority. In this study, the degrees of rejection and assimilation that different groups of people maintain regarding two types of measures designed to control the pandemic are analyzed: mandatory confinement and modification of daily routines. To do this, the emotions of a sample of 1911 people were evaluated using a questionnaire with Likert response options, an instrument constructed and validated for this research. The results allowed the identification of six emotional profiles with different sociodemographic conditions. In a pole, characterized by experiences of anger, sadness, and distrust in the face of the measures, there is a profile made up of young women, students and with a low level of economic income. At the other pole, characterized by presenting a state of comfort in the face of the pandemic and confidence in the proposed measures, there is a group made up of men, aged between 40 and 69 years, with a high educational level and high economic income. In between, we find four more profiles, with different emotional characteristics and sociodemographic conditions. The results are discussed considering their contribution to the design of non- "homogenizing" public policies for the control of the pandemic.

Keywords: COVID-19; emotions, pandemic; public policies; lockdown
Conflicto de intereses

Los autores declaran que no existe conflicto de interés posible.

\section{Financiamiento}

No existió asistencia financiera de partes externas al presente artículo. Agradecimiento

A María Josefa Pacheco por el valiosísimo apoyo en la realización del estudio sin el cual este artículo no sería posible.

Nota

El artículo no se desprende de un trabajo anterior.

ENTIDAD EDITORA

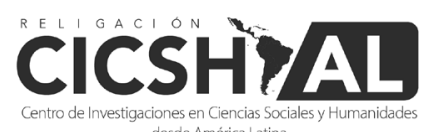




\section{Introducción}

Nos encontramos frente a la mayor crisis sanitaria que ha vivido el mundo en los últimos cien años (Ponce de León, 2020). La pandemia COVID-19 ha significado un profundo impacto en nuestra sociedad, tanto por el sufrimiento y la muerte intrínsecamente asociadas a la enfermedad, así como por el deterioro que, indirectamente, ha producido en la economía (United Nations, 2020) y la salud mental de las personas (Brooks et al., 2020; Salari et al., 2020; Sandín y Chorot, 2017). Además, en el caso de Chile en particular, la pandemia emerge sobre un trasfondo configurado por una profunda crisis sociopolítica e institucional (Fernández González y Bravo Valenzuela, 2020), por lo que no podemos hablar, en este caso, de un problema exclusivamente sanitario en un sentido estricto del término. Por esta razón, hay quienes prefieren utilizar el término "Sindemia" para referirse a la situación que atraviesa este país, denotando con ello la participación de aspectos sociales, sanitarios, económicos y de salud mental en la crisis (Jiménez et al., 2020).

Si bien es cierto que todo estado de salud o enfermedad se encuentra siempre asociado a determinantes sociales (Santos Padrón, 2011), en este caso, más que en otros, los aspectos sociales y culturales vinculados a la expresión de la pandemia cobran suma relevancia a la hora de comprender la crisis y generar políticas públicas destinadas a disminuir la propagación de la enfermedad. Además, a la espera de los efectos de inmunización que producirá la vacunación masiva de la población, las diferentes formas de contención de la pandemia que se han implementado en el mundo a través de medidas de control social, juegan, todavía, un rol central en este proceso. En este contexto, si se consideran las características de mutabilidad del virus (Ortega, 2020), algunos autores afirman que, aun cuando la vacunación generalizada y sus efectos sean una realidad, nos encontraremos conviviendo, igualmente, durante algunos años, con medidas sociales para la mitigación del contagio (Leiva y et al., 2020; Sandín et al., 2020). Ejemplos de esto último, han sido las disposiciones que apuntan a reducir o eliminar el contacto estrecho entre las personas, dado que sabemos que el principal mecanismo de transmisión de la enfermedad es a través de micro o nano aerosoles de saliva (Centro Nacional de Información en Ciencias Médicas, 2020).

Se trata, en términos generales, de un objetivo que puede alcanzarse a través de políticas públicas tanto sancionadoras como promotoras (Gómez, 2012). Respecto de las primeras, corresponden a políticas que "pretenden desestimular un tipo de comportamiento o práctica contraria a los intereses públicos y corregir el desequilibrio producido por la vulneración de la norma” (Gómez, 2012, p. 228). En el caso de la pandemia por la COVID-19, como un ejemplo de este tipo de medidas, tenemos las multas por el incumplimiento de las normas de confinamiento obligatorio, estrategia que, si bien reduce prácticamente por completo el contacto físico entre las personas, tiene diferentes efectos colaterales no deseados que deben sopesarse a la hora de decidir su aplicación. Tanto por el negativo impacto en la economía y la pérdida de empleos, el aumento de las brechas de aprendizaje producto del cierre temporal de establecimientos educacionales, $y$, sobre todo, por el impacto en la salud mental de los habitantes, este tipo de medidas se transforman en políticas de carácter extremo, difícilmente sostenibles de forma continuada en el tiempo (Verdugo, 2021). Relacionado con este último punto, Guenther (2013), analiza los efectos del confinamiento del sistema carcelario de EE. UU. y describe los devastadores 
resultados que esta práctica produce debido al carácter intrínsecamente intersubjetivo de la psicología humana. En esta misma línea, De Jaegher (2019) afirma que se trata de una medida que rompe la organicidad de la sociedad y produce riesgos que afectan su existencia en un sentido epistemológico, ontológico y ético.

Por otra parte, están las políticas promotoras, que refieren a directrices que incentivan "un tipo específico de actuación considerado conveniente para resolver problemas de interés público" (Gómez, 2012, p. 227). Como ejemplo de éstas, en relación con la pandemia, se encuentran todas aquellas regulaciones que buscan la promoción de un cambio en las rutinas diarias de la población con el objetivo de minimizar los riesgos de contagio de la enfermedad. Algunas de ellas son el uso de mascarillas o tapabocas, el lavado frecuente de manos y la mantención de una mayor distancia física durante la interacción entre personas (Organización Mundial de la Salud [OMS], 2020). A pesar de que estas medidas disminuyen prácticamente a cero el surgimiento de efectos colaterales indeseados (como los anteriormente señalados a propósito de las políticas sancionatorias), existen, no obstante, otro tipo de dificultades asociadas a estas normas. A diferencia de los confinamientos obligatorios, cuya implementación puede controlarse de forma principalmente punitiva a través de fiscalización y multas en caso de incumplimiento, cuando se trata de la promoción de medidas consistentes en el cambio de rutinas, éste no puede ser el mecanismo principal para su realización. Lo anterior, puesto que resulta físicamente imposible contar con los recursos tecnológicos y humanos necesarios para controlar su acatamiento entre todos los habitantes de una población determinada, ya que debiésemos tener un policía en cada lugar donde se produce interacción entre personas, controlando el uso de tapabocas o el lavado de manos, por ejemplo. En esta situación, se requieren mecanismos de promoción que operen sobre el desarrollo de conciencia individual y colectiva respecto de los riesgos asociados a la enfermedad y sus mecanismos de contagio.

Aspectos como los anteriormente analizados han significado que, durante este tiempo de pandemia, los países se hayan diferenciado unos de otros según el grado en el cual han optado preferentemente por la adopción de medidas más radicales y restrictivas como los confinamientos obligatorios, asumiendo de diferentes formas los costos económicos, políticos y psicosociales implicados, o, por el contrario, se hayan inclinado, principalmente, por medidas del segundo tipo, intentando promover un cambio conductual y consciente entre sus habitantes (para una revisión sobre las estrategias implementadas en el mundo frente a la pandemia COVID-19 y las lecciones que de éstas pueden sacarse ver Pemandu y Delivery, 2020). Por supuesto, no se trata de determinaciones incompatibles las unas con las otras, sino que, por el contrario, tal vez ocurra que las políticas más eficaces se encuentren en una combinación de ambos tipos de medidas.

\subsection{Emociones y política pública}

Sin embargo, más allá de cuál sea la estrategia específica que se adopte, para su correcta implementación se requiere contar con una apropiada disposición a acatar las medidas por parte de la población, un problema que pasa, en gran parte, por el acoplamiento afectivo emocional entre los ciudadanos y la política pública que se desarrolle. En palabras de Podestá (2001): 
En la formulación de políticas debe existir una fase asociada a la difusión, cuyo objetivo sea vincular al Estado con los ciudadanos en una dimensión no sólo racional, sino también afectiva. (...) sólo de esa manera podremos hablar que una política se legitima en la población, y es asumida y encarnada por los ciudadanos (Podestá 2001, p. 170).

Así, cuando hablamos de la posición que las personas expresan frente a las medidas que se proponen para encauzar un cambio social, estamos aludiendo a un problema de características emocionales. Por esta razón, el cumplir o no con las medidas señaladas no corresponde a un tipo de resolución estrictamente cognitiva, en el sentido más restringido del término, es decir, excluyendo la participación de las emociones en este proceso (Bächler, 2019). Por el contrario, como bien ha demostrado el neurocientífico portugués, Antonio Damasio, los afectos, bajo la forma de marcadores somáticos, participan directamente en la toma de decisiones, adelantándose a los procesos conscientes o más explícitos involucrados (Damasio, 2009). Desde este punto de vista, las emociones constituyen evaluaciones implícitas de la realidad que nos proveen de información acerca del mundo por lo que tienen un carácter intrínsecamente cognitivo (Solomon, 2007). Siguiendo esta misma línea de argumentación, un constructo que resulta útil para comprender el posicionamiento que las personas presentan respecto de la política pública es el de "actitudes", entendidas como tendencias psicológicas que se expresan al evaluar una entidad en particular con cierto grado de aprobación o desaprobación (Albarracin et al., 2005). Las actitudes son estados que tienen una triple dimensión, cognitiva, afectiva y conductual, respecto de los cuales, el componente evaluativo reside en la dimensión emocional de estas, y de forma más específica, en la valencia e intensidad de los afectos. De esta forma, las emociones, y más específicamente el componente afectivo de las mismas constituye un elemento central para el desarrollo de las actitudes ya que la evaluación encarnada en el afecto puede condicionarse, asociarse, inferirse, atribuirse o transformarse de otro modo en las tendencias evaluativas de actitud (Clore \& Schnall, 2005).

Asumiendo esta concepción, si lo que se desea es conocer la posición que adopta la población frente a una política pública, indagar en las emociones que se experimentan constituye una vía adecuada para conocer cuál es la perspectiva más profunda o implícita que las personas mantienen respecto del tema. Además, esta estrategia puede resultar más fidedigna que el consultar por las opiniones que se mantienen sobre la política pública, dado el efecto que tiene la deseabilidad social frente a esta forma de indagación (Domínguez et al., 2012), así como, respecto de la conveniencia de distinguir niveles más y menos implícitos para conocer la disposición que tienen las personas respecto de un tema (Pozo et al., 2006). Además, lejos de la visión de sentido común que impera en nuestra cultura dualista, las emociones no son aspectos que se limitan a acompañar la cognición, influyendo o distorsionando el pensamiento. Por el contrario, varios son los autores que reconocen un carácter intrínsecamente cognitivo en los afectos (De Sousa, 1987; Nussbaum, 2008; Solomon, 2007).

Antecedentes como los mencionados sugieren la conveniencia de evaluar las emociones que se experimentan frente a la política pública destinada al control de la pandemia COVID-19, con el objetivo de interpretarlas como indicadores de la disposición que las personas tienen en el sentido de cumplir o no con las medidas. Asumir este enfoque implica la necesidad de definir 
cuáles son las emociones más adecuadas para una investigación de estas características. Sobre este punto, en este estudio se decidió considerar el enojo, la tristeza y la confianza, como las emociones más pertinentes para realizar la evaluación, entendiendo que cada una de ellas puede ser concebida como una forma de evaluación implícita de la realidad (Ortony \& Clore, 2015). Desde esta perspectiva, puede entenderse el enojo como una emoción que nos habla de la percepción de desaire e injusticia y que sugiere, en consecuencia, una tendencia a resistirse o, incluso, a buscar cambiar el estado de las cosas (Solomon, 2007). Respecto de la tristeza, se trata de una emoción que puede ser considerada como un afecto que, aun cuando es también de valencia negativa, como en el caso de la rabia, implica procesos de reflexión interna que llevan a la introversión más que a una actuación o resistencia respecto del mundo externo (Choliz, 2005). Finalmente, respecto de la confianza, hemos asumido esta emoción como la base de la cooperación y organización social (Barbalet, 1996), un estado psicológico basado en la aceptación de la propia vulnerabilidad y que conlleva expectativas positivas respecto de las intenciones y el comportamiento de otros (Rousseau et al., 1998).

\subsection{Emociones en diferentes grupos sociodemográficos}

Además de lo anterior, es importante tener en cuenta que para una adecuada evaluación de las emociones que las personas experimentan frente a las medidas establecidas para el control de la pandemia, es necesario considerar que dichos estados son modulados por la identidad personal y los significados que se atribuyen a una situación determinada (Barrett, 2018). Por esta razón, es probable que los afectos no se manifiesten del mismo modo entre diferentes grupos con características sociodemográficas específicas. Así, por ejemplo, podemos suponer que aquellas medidas que implican distanciamiento físico entre las personas, o, incluso, una supresión total de la interacción, encontrarán mayor resistencia entre personas más jóvenes. Lo anterior, puesto que este grupo de la población se encuentra en proceso de construcción social de su identidad y asigna, por lo mismo, una mayor importancia a la interacción con sus pares (Emerson, 2020). De hecho, algunos estudios realizados durante este tiempo de pandemia refieren que las personas jóvenes (entre 18 y 30 años) exhibieron mayores niveles de angustia por los estados de confinamiento decretados (Mazza et al., 2020), experiencia que pudiera ser motivo de resistencia en el futuro frente a este tipo de medidas, o incluso, una negación de estas.

Por otra parte, tanto debido a las diferencias emocionales específicas que existen entre hombres y mujeres (Comisión Interamericana de Mujeres, 2020), así como, por factores socioculturales que significan un recargo de responsabilidades para las mujeres en condiciones de confinamiento (Apaza et al., 2020), es probable que ellas experimenten con mayor intensidad que los hombres las emociones de valencia negativa que pudieran surgir frente al confinamiento y la modificación de las rutinas. De hecho, diferentes estudios realizados recientemente encontraron que las mujeres resultaron más afectadas por la pandemia que los hombres, en un sentido psicológicamente negativo (Cuiyan et al., 2020).

Además, es posible que algunos aspectos asociados a las condiciones laborales de las personas, e incluso su nivel de ingresos económicos, sean factores que se relacionen con la disposición 
a acatar medidas de este tipo. Lo anterior, dado que es probable que determinados grupos de personas no se encuentren en condiciones de cumplir con normativas de confinamiento obligatorio, debido a la precariedad laboral en la cual se sitúan, lo que los obliga a salir a la calle a buscar formas de obtener ingresos (Coates et al., 2020; Johnsonn et al., 2020). Finalmente, aun cuando se trata de un asunto controvertido (Hawryluck et al., 2004) algunos estudios empíricos han hallado que mientras más bajo sea el nivel de educación formal que tienen las personas, mayor impacto psicológico producen los estados de confinamiento (Taylor et al., 2008).

Es posible que algunas de las condiciones sociodemográficas analizadas sean factores que predispongan negativamente a parte de la población frente a la adopción de las medidas de control de la pandemia en caso de vernos enfrentados a nuevas olas, rebrotes, o el surgimiento de nuevas cepas de la enfermedad. Además, estas condiciones pudieran tener más efecto en una nueva oportunidad ya que la evidencia refiere que la exposición acumulada a situaciones de estrés, como pueden ser el confinamiento o la modificación de las rutinas, provoca conductas reactivas, pérdida de la armonía y experiencias perturbadoras (Truco, 2002). Esto es lo que investigaciones recientes han denominado como "fatiga pandémica", es decir "desmotivación para seguir comportamientos protectores recomendados, que emerge gradualmente con el tiempo y que se ve afectada por una serie de emociones, experiencias y percepciones" (World Health Organization, 2020, p. 4, la traducción es nuestra).

Todo lo anterior invita a preguntarse ¿cuáles son las emociones más y menos intensamente experimentadas frente a las medidas de control de propagación de la pandemia provocada por la COVID-19? A su vez, parece relevante indagar también por la factibilidad de identificar perfiles a partir de la evaluación de emociones y si estos se relacionan de algún modo con distintas condiciones sociodemográficas.

Contar con información acerca de las características emocionales y sociodemográficas de dichos grupos aportaría información para diseñar estrategias de control y promoción de conductas diferenciadas y ad hoc para cada uno de ellos, avanzando así, desde una política pública homogénea como respuesta a la pandemia, hacia la necesaria atención de la diversidad psicosocial en su implementación.

Para responder a las preguntas anteriores, este estudio se propuso evaluar las emociones que experimentan las personas frente a las medidas de confinamiento obligatorio y de modificación de rutinas en la vida cotidiana identificando perfiles emocionales susceptibles de ser relacionados con distintas condiciones sociodemográficas de la muestra de participantes.

\section{Método}

El estudio es de nivel exploratorio descriptivo-correlacional y recurrió a metodologías cuantitativas para las fases de levantamiento y análisis de datos (Johnson et al., 2007). El muestreo fue no probabilístico de tipo bola de nieve y se utilizaron plataformas online para contactar a los participantes y para la posterior aplicación del instrumento. La técnica de muestreo estuvo orientada a obtener participantes con diferentes ocupaciones (estudiantes, trabajadores, jubilados/pensionados, personas dedicadas a labores domésticas y desempleados); género y rangos etarios. 


\subsection{Participantes}

Para asegurar una adecuada diversificación de la muestra en los términos antes señalados, el instrumento se distribuyó a través de instituciones públicas y privadas (municipios, universidades, empresas y ONGs) ubicadas en las zonas de mayor densidad del país (Región Metropolitana de Santiago de Chile, Región del Bío Bío y Región de Valparaíso). Este proceso se llevó a cabo previa gestión de autorización con las distintas instituciones.

La muestra final quedó conformada por 1911 participantes y el levantamiento de datos se realizó entre los meses de julio y agosto de 2020, periodo en que el país se encontraba en estado de emergencia y con confinamientos obligatorios en gran parte del territorio nacional. Por otra parte, de acuerdo con los datos del Departamento de Estadística e Información en Salud el país registraba en ese momento 1732 muertes por Covid-19. La Tabla 1 resume las características sociodemográficas de la muestra.

Tabla 1. Características de la Muestra del Estudio

\begin{tabular}{|c|c|c|c|}
\hline Variables & Categoría & Frecuencia & Porcentaje \\
\hline \multirow[t]{4}{*}{ Edad } & Entre 18 y 25 años & 702 & $36,9 \%$ \\
\hline & Entre 26 y 39 años & 559 & $29,4 \%$ \\
\hline & Entre 40 y 54 años & 442 & $23,3 \%$ \\
\hline & Más 55 años & 208 & $10,4 \%$ \\
\hline \multirow[t]{3}{*}{ Genero } & Masculino & 476 & $24,9 \%$ \\
\hline & Femenino & 1423 & 74,5 \\
\hline & Otro & 12 & $0,6 \%$ \\
\hline \multirow[t]{6}{*}{ Actividad } & Labores domésticas & 50 & $2,6 \%$ \\
\hline & Trabajadores dependientes & 841 & $44,0 \%$ \\
\hline & Trabajadores independientes & 157 & $8,2 \%$ \\
\hline & Estudiantes & 706 & 36,9 \\
\hline & Jubilados o pensionados & 42 & $2,2 \%$ \\
\hline & Desempleados & 93 & 4,9 \\
\hline \multirow[t]{8}{*}{ Ingresos USD } & Sin ingresos & 622 & $32,5 \%$ \\
\hline & $\begin{array}{c}\text { Menos de } \\
380\end{array}$ & 202 & $10,6 \%$ \\
\hline & $\begin{array}{c}\text { Entre } 380 \\
\text { y } 660\end{array}$ & 202 & $10,6 \%$ \\
\hline & $\begin{array}{c}\text { Entre } 660 \\
\text { y } 1056\end{array}$ & 272 & $14,2 \%$ \\
\hline & $\begin{array}{c}\text { Entre } 1056 \\
\text { y } 1598\end{array}$ & 258 & $13,5 \%$ \\
\hline & $\begin{array}{c}\text { Entre } 1598 \\
\text { y } 2333\end{array}$ & 185 & $9,7 \%$ \\
\hline & $\begin{array}{c}\text { Entre } 2333 \\
\text { y } 3218\end{array}$ & 80 & $4,3 \%$ \\
\hline & Más de 3218 & 90 & $4,7 \%$ \\
\hline \multirow[t]{5}{*}{ Estudios } & Educación básica o primaria & 13 & $0,7 \%$ \\
\hline & Educación media o secundaria & 647 & $33,9 \%$ \\
\hline & Educación Técnica & 137 & $7,2 \%$ \\
\hline & Educación Universitaria o Superior & 694 & $36,3 \%$ \\
\hline & Educación de Postgrado & 419 & $21,9 \%$ \\
\hline
\end{tabular}

Fuente: elaborado por los autores 


\subsection{Técnicas e Instrumentos}

Para la recolección de datos se construyó un instrumento que fue denominado "Escala para la evaluación de las emociones frente a la crisis socio-sanitaria provocada por la pandemia Covid-19". Este instrumento contenía opciones de respuesta tipo Likert, y su aplicación fue realizada bajo la modalidad de auto reporte online. El instrumento fue elaborado para realizar una serie de estudios con objetivos y alcances diferentes por lo que su diseño contempló más aspectos que los que fueron incluidos en la presente investigación, tal y como se explicitará más adelante. El instrumento completo permite evaluar la intensidad de siete emociones (enojo o rabia, temor o miedo, repugnancia o asco, interés o entusiasmo, alegría o felicidad, confianza o aceptación y tristeza o pena) considerando las siguientes opciones de respuesta: a) No he sentido esta emoción (puntuado con un punto); b) La he sentido con baja intensidad (puntuado con dos puntos); c) La he sentido con mediana intensidad (puntuado con tres puntos); d) La he sentido con alta intensidad (puntuado con cuatro puntos); e) La he sentido con muy alta intensidad (puntuado con cinco puntos).

Teniendo en cuenta la complejidad de la crisis socio-sanitaria descrita en la fundamentación de este trabajo, el instrumento evalúa cada una de las emociones anteriores respecto de las siguientes ocho dimensiones: a) Obligación de mantener distanciamiento físico entre las personas; b) Obligación de modificar las rutinas diarias producto de la pandemia; c) Situación económica del país; d) Posibilidad de contagio de la enfermedad; e) Manejo del gobierno respecto de la situación socio-sanitaria; f) Posibilidad de que se retomen las actividades normales; g) Interferencia que la pandemia ha significado para los procesos de cambio social que se estaban produciendo en el país; h) Obligación de mantener estados de cuarentena o confinamiento obligatorios.

En función de los objetivos del presente estudio, el diseño metodológico consideró la evaluación de tres emociones: rabia, tristeza y confianza, expresadas respecto de dos dimensiones de la crisis socio-sanitaria: "modificación de las rutinas diarias" y "estados de cuarentena o confinamiento obligatorio". La Figura 1 muestra la estructura de los ítems:

Figura 1. Estructura de los ítems del instrumento

\begin{tabular}{|l|l|l|l|l|l|}
\cline { 2 - 5 } \multicolumn{1}{c|}{} & $\begin{array}{l}\text { No he senti- } \\
\text { do esa emo- } \\
\text { ción }\end{array}$ & $\begin{array}{l}\text { La he sentido } \\
\text { con baja in- } \\
\text { tensidad }\end{array}$ & $\begin{array}{l}\text { La he sentido } \\
\text { con mediana } \\
\text { intensidad }\end{array}$ & $\begin{array}{l}\text { La he sentido } \\
\text { con alta in- } \\
\text { tensidad }\end{array}$ & $\begin{array}{l}\text { La he sentido } \\
\text { con muy alta } \\
\text { intensidad }\end{array}$ \\
\hline $\begin{array}{l}\text { Frente a la obligación de modificar las rutinas } \\
\text { diarias producto de la pandemia, he sentido ra- } \\
\text { bia }\end{array}$ & & & & & \\
\hline $\begin{array}{l}\text { Frente a la obligación de modificar las rutinas } \\
\text { diarias producto de la pandemia, he sentido tris- } \\
\text { teza }\end{array}$ & & & & & \\
\hline $\begin{array}{l}\text { Frente a la obligación de modificar las rutinas } \\
\text { diarias producto de la pandemia, he sentido } \\
\text { confianza }\end{array}$ & & & & & \\
\hline $\begin{array}{l}\text { Frente a los estados de cuarentena o confina- } \\
\text { miento que se desarrollan en el país, he sentido } \\
\text { rabia }\end{array}$ & & & & & \\
\hline $\begin{array}{l}\text { Frente a los estados de cuarentena o confina- } \\
\text { miento que se desarrollan en el país, he sentido } \\
\text { tristeza }\end{array}$ & & & & & \\
\hline $\begin{array}{l}\text { Frente a los estados de cuarentena o confina- } \\
\text { miento que se desarrollan en el país, he sentido } \\
\text { confianza }\end{array}$ & & & & & \\
\hline
\end{tabular}

Fuente: elaborado por los autores 
El instrumento se sometió a un proceso de validación de contenido y fiabilidad. Para la validez de contenido se utilizó la metodología panel de expertos $(\mathrm{N}=10)$ el cual se conformó por un cuoteo de profesionales de las ciencias sociales del ámbito académico, investigación e intervención directa con personas. A su vez se utilizó la metodología V de Aiken (1985) la que permite obtener información sobre la proporción de jueces que manifiestan una apreciación positiva sobre el objeto valorado y que puede adoptarse como criterio para tomar decisiones en cuanto a la pertinencia de revisar o eliminar ítems. Para la aplicación de esta metodología se formularon dos preguntas (escala tipo Likert) respecto a la claridad y pertinencia de los enunciados de cada uno de los ítems. Se estableció como criterio para la eliminación de ítems y/o revisión de estos para su modificación, valores $\mathrm{V}$ de Aiken inferiores a 0.7 (equivalentes a puntuaciones medias de la escala tipo Likert inferiores a 3). El resultado obtenido fue superior a .8 Para el análisis de fiabilidad se utilizó el alfa de Cronbach alcanzando un valor de .951

\subsection{Consideraciones éticas}

El estudio se realizó siguiendo las reglas de la Declaración de Helsinki de 1975 y revisada en 2013, contando además con la aprobación del Comité Ético Científico de la Universidad Mayor Chile, bajo resolución C.R.I. 245, institución patrocinadora de esta investigación. Así mismo, se aplicó un formulario de consentimiento informado con el objetivo de garantizar el tratamiento anonimizado de los datos, la confidencialidad en el uso de la información y su utilización con fines de investigación exclusivamente, así como la total libertad de los participantes para abandonar el proceso cuando lo quisieran.

\subsection{Procedimientos}

El procesamiento y el análisis de datos se realizó a través del software estadístico SPSS versión 24. En primer lugar, se hicieron análisis descriptivos y comparación de medias, con el propósito de caracterizar a los participantes en función de la intensidad con la cual experimentaban cada una de las emociones frente a las medidas de control de la pandemia. Para realizar los análisis anteriores se utilizó una prueba no paramétrica, específicamente el estadístico $U$ de MannWhitney, dado que se observó un comportamiento atípico en la muestra ( $p>.05)$.

En segundo lugar, se aplicó una técnica multivariante, particularmente, análisis de clústeres no jerárquicos con el propósito de explorar la existencia de conglomerados diferenciados. Cabe señalar que los clústeres o conglomerados tienen el propósito de agrupar a individuos que poseen características comunes y diferenciadas de otros grupos. En este estudio, el proceso de conformación de clústeres se realizó considerando como variable de entrada la intensidad con la cual los participantes referían haber experimentado las emociones estudiadas (enojo, tristeza y confianza) en relación con cada una de las dos dimensiones de evaluación ("modificación de las rutinas diarias" y “estados de cuarentena o confinamiento obligatorio"). Para definir la cantidad de clústeres a analizar se consideraron diferentes cantidades de agrupación (desde tres hasta diez conglomerados) cada uno de los cuales fue examinado en función de dos diferentes criterios. En primer lugar, se tuvo en cuenta que en cada uno de los grupos resultantes hubiese al menos una emoción con una intensidad por sobre 3.0 (en una escala Likert que iba de 1 a 5 ) 
vale decir, de mediana a muy alta intensidad. En segundo lugar, se consideró que las emociones que presentaban la condición anterior en cada grupo lo hiciesen respecto de dimensiones de evaluación diferentes de forma de poder identificar diferencias entre los grupos.

Para la ejecución de este análisis se utilizó la técnica de clustering denominada K-means, procedimiento inductivo de clasificación que consiste en una división de unidades a clasificar en un número predeterminado de clúster y, a partir de la misma, realizar una serie de movimientos hasta encontrar el resultado que minimice las diferencias intragrupales y maximice las diferencias intergrupales

Una vez definidos los clústeres se examinó la relación entre los perfiles y las diferentes variables sociodemográficas (género, edad, ocupación, nivel de ingresos económicos y nivel educacional) a través de chi cuadrado y residuos tipificados corregidos. Por último, se procedió a la descripción de estos en términos de perfiles emocionales respecto de las medidas para el control de la pandemia.

\section{Resultados}

Figura 2. Intensidad de las emociones frente a la modificación de las rutinas diarias

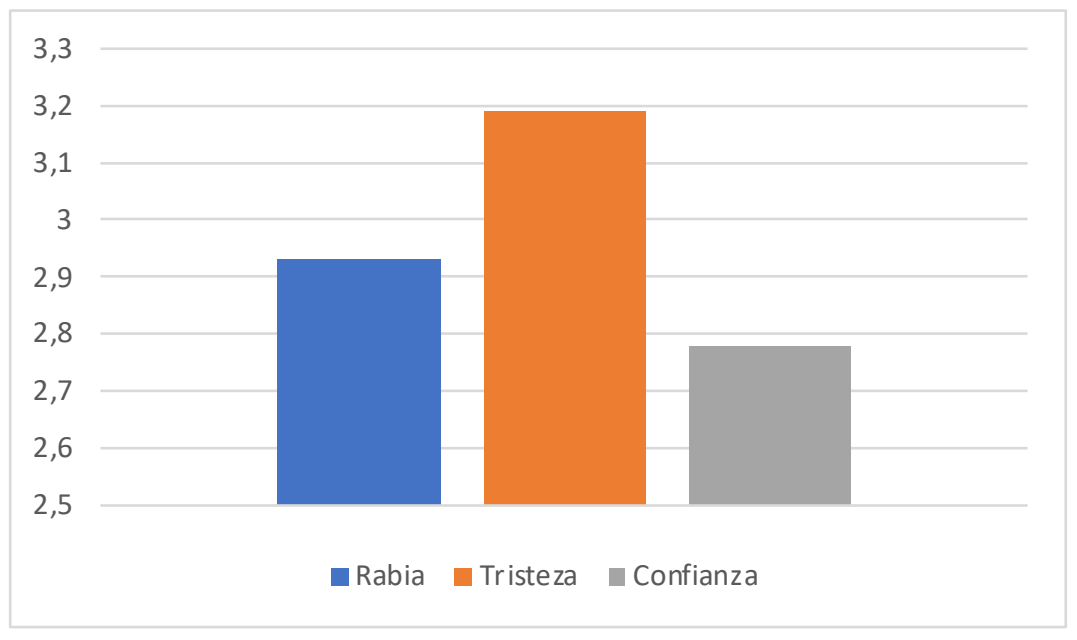

Fuente: elaborada por los autores

Una vez constatada la existencia de diferencias entre las tres emociones evaluadas $(p<.05)$, puede afirmarse que la emoción más intensamente experimentada por los participantes en el estudio frente a la dimensión “Modificación de las rutinas diarias" fue la tristeza (X=3.19), seguida en segundo lugar, del enojo $(X=2.93)$. Por otro lado, la emoción menos intensamente experimentada en esta dimensión fue la confianza $(X=2.78)$. 
Figura 3. Intensidad de las emociones frente a los estados de cuarentena o confinamiento obligatorio

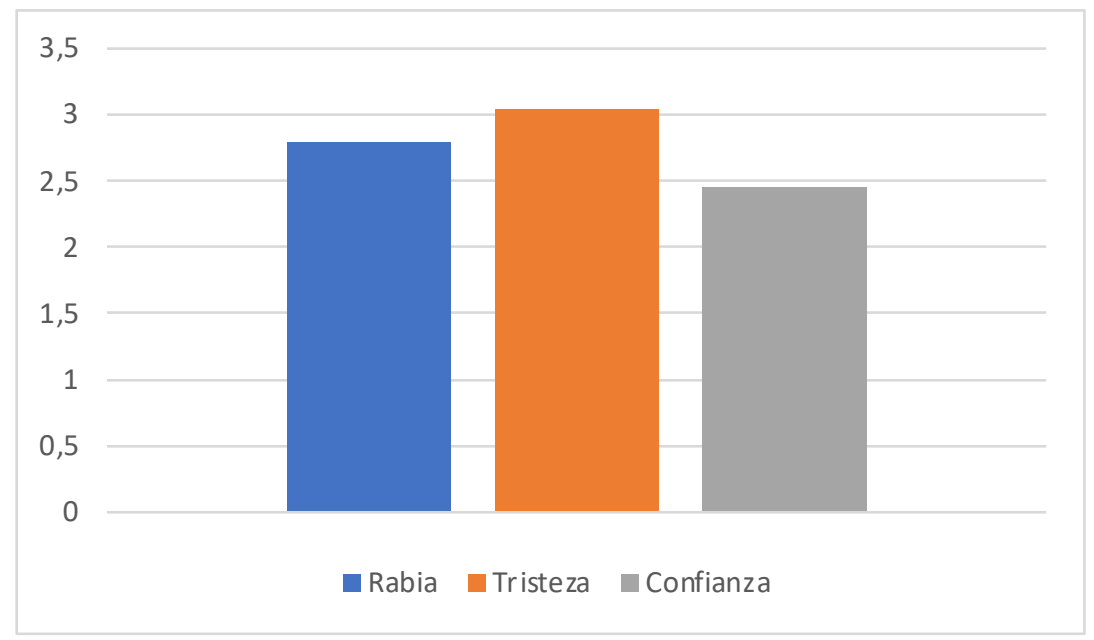

Fuente: elaborada por los autores

Al igual que en el caso anterior, una vez constatada la existencia de diferencias entre las tres emociones evaluadas ( $\mathrm{p}<.05$ ), puede afirmarse, que la emoción más intensamente experimentada por los participantes en el estudio frente a la dimensión "Estados de cuarentena o confinamiento obligatorio" fue la tristeza $(X=3.04)$, seguida, en segundo lugar, del enojo $(X=2.79)$. Por otro lado, en este caso, la emoción menos intensamente experimentada frente a esta dimensión fue, también, la confianza $(X=2.45)$.

Los resultados anteriores nos muestran que las personas se encuentran, en general, entristecidas frente a las medidas establecidas para el control de la pandemia (con una intensidad media), emoción que se acompaña de enojo frente a las mismas. Por otra parte, en coherencia con esta experiencia de desagrado, los resultados obtenidos dan cuenta, también, de un bajo grado de confianza frente a ambos tipos de medidas implementadas: cuarentanas obligatorias y modificación de las rutinas. No obstante, para una mejor comprensión de la experiencia de las personas frente a la pandemia, resulta necesario profundizar en el análisis mediante la identificación de perfiles emocionales específicos, indagando, además, posibles relaciones entre éstos y diferentes condiciones sociodemográficos.

Para cumplir con el objetivo anterior, se realizó un análisis de conglomerados o clústeres de $K$ medias. Tal y como se explicó previamente, el análisis se realizó a partir de los resultados obtenidos para las dimensiones "Emociones frente a los estados de cuarentena o confinamiento" y "Emociones frente a la modificación de las rutinas diarias". Teniendo en cuenta los criterios especificados en el apartado de procedimientos, se consideraron distintas posibilidades en función del número de clústeres a incluir en el análisis, optándose, finalmente, por una clasificación en seis grupos, puesto que se consideró que esta cantidad permitía describir de mejor forma diferentes perfiles emocionales.

La Tabla 2 muestra los diferentes grupos obtenidos los cuales se diferencian entre sí por el grado de intensidad emocional que presentaron en cada una de las dimensiones evaluadas. 
Los valores resaltados con color gris indican aquellas medias de emociones que tienen mayor intensidad en cada grupo (sobre 3.0).

Tabla 2. Clústeres resultantes de la clasificación a partir de la intensidad de las emociones

\begin{tabular}{|c|c|c|c|c|c|c|}
\hline & $\begin{array}{c}\text { Clúster } \\
1 \\
\text { (389 personas) }\end{array}$ & $\begin{array}{c}\text { Clúster } \\
2 \\
\text { (243 personas) }\end{array}$ & $\begin{array}{c}\text { Clúster } \\
3 \\
\text { (307 personas) }\end{array}$ & $\begin{array}{c}\text { Clúster } \\
4 \\
\text { (331 personas) }\end{array}$ & $\begin{array}{c}\text { Clúster } \\
5 \\
\text { (280 personas) }\end{array}$ & $\begin{array}{c}\text { Clúster } \\
6 \\
(361 \text { personas) }\end{array}$ \\
\hline $\begin{array}{l}\text { Enojo frente al } \\
\text { confinamiento }\end{array}$ & 4.46 & 3.32 & 1.66 & 1.83 & 4.05 & 1.50 \\
\hline $\begin{array}{l}\text { Tristeza frente al } \\
\text { confinamiento }\end{array}$ & 4.54 & 2.17 & 2.02 & 3.43 & 4.16 & 1.67 \\
\hline $\begin{array}{l}\text { Enojo frente a la } \\
\text { modificación de } \\
\text { las rutinas }\end{array}$ & 4.35 & 3.77 & 2.02 & 3.57 & 2.13 & 1.63 \\
\hline $\begin{array}{l}\text { Tristeza frente a } \\
\text { la modificación } \\
\text { de las rutinas }\end{array}$ & 4.49 & 2.98 & 2.44 & 4.19 & 2.92 & 1.85 \\
\hline $\begin{array}{l}\text { Confianza frente } \\
\text { al confinamiento }\end{array}$ & 1.76 & 2.13 & 1.47 & 3.35 & 2.49 & 3.37 \\
\hline $\begin{array}{l}\text { Confianza frente } \\
\text { a la modificación } \\
\text { de las rutinas }\end{array}$ & 2.24 & 2.68 & 1.89 & 2.85 & 3.23 & 3.75 \\
\hline
\end{tabular}

Fuente: elaborada por los autores

Los resultados dan cuenta de la existencia de seis grupos con características emocionales claramente diferenciadas. En términos generales, puede decirse que al lado izquierdo de la Tabla 2 se encuentran aquellos grupos que presentan únicamente experiencias emocionales de valencia negativa frente a las medidas para el control de la pandemia (clústeres 1 y 2 ), mientras que, hacia el lado derecho, se ubican aquellos grupos que tienen mayor confianza frente a las medidas implementadas (clústeres 4, 5 y 6). En medio, se observa un grupo que presenta una baja intensidad en todas las emociones evaluadas (clúster 3 ).

Por otro lado, con el fin de examinar las características sociodemográficas que predominan en los integrantes de cada grupo, se realizó un análisis de $X^{2}$. La Tabla 3 expone los resultados de este análisis, el cual permite afirmar que cada uno de los conglomerados posee características sociodemográficas distintivas.

Tabla 3. Relación entre los conglomerados y diferentes condiciones sociodemográficas

\begin{tabular}{|c|c|c|c|c|}
\hline EDAD & GÉNERO & OCUPACIÓN & INGRESOS & ESTUDIOS \\
\hline$\chi^{2}=105,126 ;$ & $\chi^{2}=39,723 ;$ & $\chi^{2}=106,190 ;$ & $\chi^{2}=105,591 ;$ & $\chi^{2}=85,473 ;$ \\
$g \mid=25 ;$ & $g \mid=10 ;$ & $g \mid=30 ;$ & $g \mid=35 ;$ & $g \mid=20 ;$ \\
$p<, 001$ & $p<, 001$ & $p<, 001$ & $p<, 001$ & $p<, 001$ \\
\hline
\end{tabular}

Fuente: elaborada por los autores

Los hallazgos anteriores sugieren la conveniencia de realizar un análisis de residuos tipificados corregidos considerando las variables sociodemográficas incluidas en el estudio, con el objetivo de conocer cómo se distribuyen estas características. Las Tablas 4 y 5 presentan los resultados de este análisis destacándose en color gris las tendencias significativas en cada grupo. 
Tabla 4. Análisis de residuos tipificados corregidos para la edad, el género y el tipo de actividad realizada

\begin{tabular}{|c|c|c|c|c|c|c|c|}
\hline & Categorías & $\begin{array}{c}\text { Clúster } \\
1\end{array}$ & $\begin{array}{c}\text { Clúster } \\
2\end{array}$ & $\begin{array}{c}\text { Clúster } \\
3\end{array}$ & $\begin{array}{c}\text { Clúster } \\
4\end{array}$ & $\begin{array}{c}\text { Clúster } \\
5\end{array}$ & $\begin{array}{c}\text { Clúster } \\
6\end{array}$ \\
\hline \multirow{5}{*}{ Edad } & 18-25 años & 6.1 & .2 & 1.2 & .4 & -3.0 & -5.3 \\
\hline & 26-39 años & -.6 & .6 & -.6 & 1.4 & .5 & -1.2 \\
\hline & 40-54 años & -3.6 & -.6 & -1.3 & -.7 & 2.4 & 3.9 \\
\hline & $55-69$ años & -3.3 & .1 & -.3 & -1.6 & .7 & 4.5 \\
\hline & $70-85$ años & -1.7 & -.8 & 2.5 & -1.4 & .0 & 1.4 \\
\hline \multirow{2}{*}{ Género } & Femenino & 3.4 & -1.9 & -1.8 & 2.4 & 1.1 & -3.4 \\
\hline & Masculino & -3.6 & 1.9 & 2.0 & -2.7 & -1.0 & 3.7 \\
\hline \multirow{6}{*}{ Actividad } & $\begin{array}{c}\text { Labores domés- } \\
\text { ticas }\end{array}$ & -1.2 & -.8 & 2.8 & .5 & -.3 & -1.0 \\
\hline & $\begin{array}{l}\text { Trabajador } \\
\text { dependiente }\end{array}$ & -3.5 & -1.1 & -1.8 & -.1 & 3.1 & 3.6 \\
\hline & $\begin{array}{l}\text { Trabajador inde- } \\
\text { pendiente }\end{array}$ & -3.3 & 2.3 & -.5 & -1.6 & .5 & 3.1 \\
\hline & Estudiante & 6.3 & .4 & 1.1 & 1.0 & -3.8 & -5.4 \\
\hline & $\begin{array}{l}\text { Jubilado o pen- } \\
\text { sionados }\end{array}$ & -2.0 & -1.5 & 2.0 & .0 & -.4 & 1.8 \\
\hline & Desempleados & .2 & .6 & -1.2 & -.1 & 1.3 & -.7 \\
\hline
\end{tabular}

Fuente: elaborada por los autores

Tabla 5. Análisis de residuos tipificados corregidos para el nivel de ingresos y el nivel de estudios

\begin{tabular}{|c|c|c|c|c|c|c|c|}
\hline \multirow{5}{*}{ Estudios } & $\begin{array}{c}\text { Educación Bá- } \\
\text { sica }\end{array}$ & .9 & -.5 & -.8 & -.2 & 1.6 & -1.0 \\
\hline & $\begin{array}{l}\text { Ed. Media o } \\
\text { secundaria }\end{array}$ & 5.4 & -.4 & 2.0 & -.3 & -2.4 & -4.6 \\
\hline & Ed. Técnica & -.2 & -1.2 & 1.7 & .8 & .2 & -1.3 \\
\hline & Universitaria & -.7 & .9 & -1.8 & .5 & .6 & .7 \\
\hline & Postgrado & -5.4 & .3 & -1.1 & -.7 & 1.7 & 5.5 \\
\hline \multirow{8}{*}{$\begin{array}{l}\text { Ingresos } \\
\text { USD }\end{array}$} & Sin ingresos & 2,9 & 1,6 & 1,5 & ,8 & $-2,4$ & $-4,3$ \\
\hline & Menos de 380 & 3,4 &,- 9 &,- 6 &,- 5 &,- 6 & $-1,2$ \\
\hline & De 380 a 660 & $-1,2$ & $-1,2$ &, 7 &,- 7 & 2,3 & ,1 \\
\hline & De 660 a 1056 &,- 8 &,- 6 &,- 7 &,- 5 & 2,2 &, 5 \\
\hline & De 1056 a 1598 &, 5 & -6 &,- 8 & ,4 & 4 & , \\
\hline & De 1598 a 2333 & $-4,2$ & 1,7 &,- 4 &,- 2 & 1,5 & 2,0 \\
\hline & De 2333 a 3218 & $-1,8$ &,- 4 &, 7 & $-1,2$ &,- 6 & 3,2 \\
\hline & Más de 3218 & $-1,8$ &,- 7 &,- 9 & 1,4 & $-3,0$ & 4,6 \\
\hline
\end{tabular}

Fuente: elaborada por los autores

Dados los resultados obtenidos, a continuación, se presenta una descripción de cada uno de los grupos obtenidos de la clasificación, interpretando sus características como perfiles emocionales de acuerdo con la conceptualización de las emociones expuesta anteriormente. En paralelo, se describen también, las características sociodemográficas de cada perfil. 


\section{Grupo 1: Perfil “Malestar generalizado frente a la pandemia”}

Se trata del grupo más grande de todos los que surgen del análisis de clústeres (389 personas). Corresponde a un perfil emocional caracterizado por presentar una alta intensidad en las emociones de valencia negativa y una baja intensidad en las emociones de valencia positiva que consideramos en el estudio. Se trata, por tanto, de personas que se encuentran enojadas y tristes por el estado de confinamiento y la modificación de las rutinas que ha significado la pandemia, y que, consecuentemente con este estado, no confían en ninguna de estas medidas. Respecto de sus características sociodemográficas, se trata de un grupo constituido principalmente por mujeres jóvenes entre 18 y 25 años, que tienen como máximo nivel de estudios alcanzado hasta ahora la enseñanza media o secundaria, no obstante, la mayoría de ellos se encuentran actualmente estudiando. Además, se trata de personas con ingresos mensuales menores a 380 USD.

\section{Grupo 2 Perfil: “Enojo generalizado frente a la pandemia”}

Se trata del grupo más pequeño de todos los que surgen del análisis de clústeres, compuesto por 243 personas. Corresponde a un perfil emocional caracterizado por presentar una intensidad media de enojo, tanto frente la implementación de medidas de cuarentena o confinamiento, así como respecto de la modificación de las rutinas. Respecto de sus características sociodemográficas, la única característica que distingue a este grupo del resto es el hecho de que se encuentra constituido principalmente por trabajadores independientes.

\section{Grupo 3: Perfil “Aplacamiento emocional frente a la pandemia”}

Corresponde a un grupo de 307 personas, caracterizado por presentar una baja intensidad emocional en todas las emociones y dimensiones evaluadas en el estudio. En términos sociodemográficos, se trata de un perfil representado principalmente por hombres, adultos mayores, con una edad de 70 a 85 años, que tienen como máximo nivel de estudios alcanzado la enseñanza media o secundaria. Respecto de la actividad que realizan, se trata de personas que se desempeñan en labores de hogar y/o son pensionados/jubilados.

\section{Grupo 4: Perfil "Tristeza generalizada frente a la pandemia y confianza frente a las medidas} de confinamiento”

Se trata de un grupo compuesto por 331 personas. Emocionalmente, se caracterizan por presentar un alto grado de tristeza experimentada frente a la modificación de las rutinas, estado emocional que también se produce, aunque con menor intensidad, frente a la medida de confinamiento. Respecto del enojo, se trata de una emoción que sólo se presenta, en grado de intensidad medio, frente a la modificación de las rutinas. Respecto de la confianza, se presenta en un grado medio frente a la medida de confinamiento. En cuanto a sus características sociodemográficas, se trata de un perfil compuesto principalmente por mujeres. 
Grupo 5: Perfil “Malestar frente a la confinamiento y confianza frente a la modificación de las rutinas"

Corresponde a un grupo compuesto 280 personas cuyas características emocionales son el hecho de presentar tanto enojo como tristeza en un alto grado frente a la medida de confinamiento. En paralelo, presentan una confianza moderada respecto de la medida de modificación de las rutinas. Sus características sociodemográficas distintivas son el hecho de tener edades comprendidas entre los 40 y 54 años, trabajando principalmente como empleados dependientes, con una remuneración entre $\$ 380$ y $\$ 1056$ (dólares americanos).

\section{Grupo 6: Perfil “Comodidad frente a la pandemia y confianza en las medidas”}

Se trata de un grupo compuesto por 361 personas que se distinguen emocionalmente por un descenso en las emociones de valencia negativa y una confianza aumentada en ambas medidas implementadas para la contención de la pandemia (estados de confinamiento y modificación de las rutinas). Respecto de sus características sociodemográficas, se trata principalmente de hombres con edades entre los 40 y 69 años, con un nivel educacional de postgrado. Respecto de las principales ocupaciones que se asocian a este perfil, s e e ncuentran los trabajadores dependientes e independientes. Se trata del grupo con los más altos niveles de ingresos entre todos los que surgen del análisis con remuneraciones desde \$1598 USD, hacia arriba.

\section{Discusión y conclusiones}

El estudio realizado ha permitido indagar sobre la realidad bajo la cual se vive en Chile la pandemia COVID-19 desde una perspectiva diferente a las que tradicionalmente siguen este tipo de investigaciones, la mayoría de las cuales conciben las emociones como estados separados de los procesos cognitivos. En este contexto, la asunción de un enfoque no dualista conlleva tanto limitaciones como ventajas para el estudio realizado. Respecto de lo primero, la consideración de las emociones que experimenta la población como estados cognitivos impide analizar los resultados alcanzados a "la luz" de investigaciones anteriormente realizadas sobre este tema, lo cual constituye una desventaja puesto que disminuye la riqueza de los análisis que se lleven a cabo sobre los resultados obtenidos. Sin embargo, la perspectiva epistemológica asumida es a la vez una fortaleza puesto que permite aproximarse al problema de investigación considerando una indagación en niveles más implícitos del mismo, además, con una menor influencia de la deseabilidad social en el proceso. Para ello, se consideraron las emociones como indicadores de la posición que las personas adoptan frente a dos dimensiones de la política pública que se viene implementando en este país: el confinamiento obligatorio y la modificación de las rutinas diarias. La información recabada da cuenta de una sensación de tristeza generalizada entre los participantes en el estudio, emoción que se presenta con una intensidad medio alta, acompañada de enojo y confianza en un más bajo nivel de intensidad.

Este panorama general esconde, no obstante, algunas importantes diferencias emocionales existentes en grupos con características sociodemográficas particulares. El a nálisis de dichas diferencias configura un conocimiento que permite retroalimentar a los hacedores de la política 
pública contribuyendo así al desarrollo de medidas que consideren de mejor forma la diversidad en lo que respecta al control de la pandemia COVID-19, considerando matices en función de las características específicas que tienen distintos grupos de la población. Se trata de una información relevante, además, debido a que Chile, así como la mayoría de los países en el mundo, se encuentra frente a nuevos estadios de expresión de la pandemia, situación que conlleva, eventualmente, volver a implementar medidas de control en la población. Ejecutar nuevamente estas medidas, teniendo ahora a la vista este tipo de conocimientos pudiera colaborar en términos de favorecer la implementación de diferentes acentos en torno de la política pública. Así, por ejemplo, parece relevante tener en cuenta que no todas las personas tienen los mismos grados de disposición a acatar las medidas y que, incluso, existen algunos grupos que tal vez no lo hagan en ninguna circunstancia. Específicamente, el primer perfil identificado que ha sido caracterizado como "Malestar generalizado frente a la pandemia", corresponde a un grupo de personas que se encuentran muy enojadas y tristes por las medidas que se han tomado para el control de la crisis. Consecuentemente con este malestar y atendiendo a la comprensión de las emociones como estados cognitivos, planteada en la introducción de este trabajo, asi como a la definición de las emociones evaluadas, puede interpretarse que dichas personas no confían en las medidas para la mitigación del contagio y pudieran mostrarse resistentes a seguir las indicaciones derivadas de la política pública. Lamentablemente, hasta ahora, las políticas públicas implementadas no han considerado a este ni a otros grupos específicos de forma diferenciada, asumiendo, más bien, que todos los integrantes de la sociedad responderán del mismo modo frente a las medidas propuestas. Sin embargo, si se consideran las características descritas para el primer perfil identificado en este estudio, y también para el segundo, tal vez resulte adecuado privilegiar otro tipo de intervenciones con estos grupos de personas. Bajo esta lógica, si se asume que se trata de grupos que, dadas sus características intrínsecas, tenderán siempre, de forma espontánea, a resistirse frente a la imposición de medidas restrictivas, vale la pena plantearse por qué no intentar promover el cambio a través de medidas de intervención de carácter educativo antes que punitivo. En esta línea, en vez de centrar los recursos en rondas de control policial destinadas a fiscalizar el cumplimiento de las medidas, tal vez debiese contemplarse la posibilidad de realizar rondas educativas u otras formas que intervengan de forma dialogada con aquellos grupos de jóvenes que se reúnen desafiando las normativas vigentes.

Siguiendo con esta lógica de consideración de la diversidad para la implementación de la política pública, tal vez sea necesario reconocer, además, que existen importantes diferencias en los grados de adhesión de diferentes grupos de la población respecto de cada uno de los dos tipos de medidas evaluadas en este estudio: confinamiento obligatorio y cambio de rutinas. Específicamente, el cuarto perfil identificado, manifiesta más confianza en las medidas de confinamiento obligatorio, mientras que el quinto, por su parte, corresponde a uno que confía más en la modificación de las rutinas diarias como una vía para el control de la propagación de la COVID-19. No parece lógico intervenir del mismo modo con ambos grupos a través de las políticas públicas, si se consideran sus características específicas. 
Sin embargo, más allá de todo lo anterior, importante destacar que se trata de un estudio exploratorio y que la muestra evaluada no es representativa de la población chilena. Por lo mismo, los resultados deben considerarse, por ahora, como datos preliminares que requieren ser investigados con mayor profundidad, a través de futuros estudios de carácter cualitativo que permitan adentrarse con mayor detalle en las características intrínsecas de cada perfil. Esto es pertinente para todos los perfiles identificados, pero resulta especialmente necesario en el caso del tercero, denominado "Aplacamiento emocional frente a la pandemia", correspondiente a un grupo de personas que exhibe un descenso en la intensidad de todas las emociones evaluadas. La identificación de este perfil puede ser considerada como una señal de alerta sobre la posibilidad de que se trate de un grupo que dadas sus características sociodemográficas específicas (hombres, adultos mayores, con bajo nivel de estudios), se encuentre en un estado de indefensión aprendida que les dificulte reaccionar emocionalmente frente a la pandemia, o, por el contrario, que el descenso de intensidad emocional se relacione más bien con un estado de bienestar y calma subjetivos. Para dilucidar este punto, es necesario cruzar los datos de los perfiles obtenidos con una evaluación que entregue pistas acerca de la salud mental de las personas que integran cada grupo. Igualmente, parece relevante profundizar también, en la comprensión del sexto perfil identificado, denominado "Comodidad frente a la pandemia y confianza en las medidas”. Conocer con mayor detalle las características de este último grupo, pudiera entregar algunas pistas respecto de las variables que se asocian con una mejor experiencia durante la crisis socio-sanitaria y una mayor sintonía con las medidas de control de esta.

Finalmente, en la misma dirección de profundizar en los hallazgos alcanzados, cabe preguntarse por la relación existente entre los perfiles identificados y la situación sociopolítica existente en Chile al momento de realizar esta investigación. ¿Cuánto del rechazo frente a las medidas expresado por los perfiles 1 y 2 del presente estudio tiene que ver con una identificación por parte de dichos grupos respecto de los cuestionamientos que en ese momento se vertían sobre el sistema social y político en general? Una investigación más completa en el sentido anterior debiese avanzar en el análisis de la interacción entre ambos contextos (el sociopolítico y el socio-sanitario) como realidades indisociables a la hora de estudiar las vivencias de los habitantes de este país.

\section{Referencias}

Aiken, L. (1985). Three Coefficients for Analyzing the Reliability and Validity of Ratings. Educational and Psychological Measurement, 45(1), 131-42. https://doi.org/10.1177\%2Fo013164485451012

Albarracín, D., Johnson, B. T., \& Zanna, M. P. (2005). The Hanbook of Attitudes. Lawrence Erlbaum Associates, Publishers.

Apaza, C., Seminario, R., \& Santa-Cruz, J. (2020). Factores psicosociales durante el confinamiento por el Covid-19 - Perú. Revista Venezolana de Gerencia, 25(90), 402-413. https://doi.org/10.3796o/rvg. v25igo.32385 
Bächler, R. (2019). Del problema emoción-cognición a la integración de la fenomenología y la intencionalidad de los estados mentales. En P. López-Silva, Discusiones contemporáneas en Filosofía de la mente. Voces locales (pp.. 123-150). Instituto de Filosofía, Universidad de Valparaíso.

Barbalet, J. (1996). Social emotions: Confidence, trust and loyalty. International journal of sociology and social policy, 16(9/10), 7596. https://doi.org/10.1108/eb013270

Barrett, L. (2018). La vida secreta de cerebro. Como se construyen las emociones. Paidós.

Brooks, S., Webster, R., Smith, L., Woodland, L., Wessely, S., Greenberg, N., \& James, G. R. (2020). The psychological impact of quarantine and how to reduce it: rapid review of the evidence. Lancet, 395(10227). 912-920. https://doi.org/10.1016/S0140-6736(20)30460-8

Centro Nacional de Información en Ciencias Médicas. (2020). Coronavirus 2019 actualización. Centro Nacional de Información de Ciencias Médicas/INFOMED. https://temas.sld.cu/coronavirus/2019ncov/actualización17defebrerode2020

Charland, L. C. (2005). The Heat of Emotion: Valence and the demarcation problem. Journal of Consciousness Studies, 12(8), 82-102.

Choliz, M. (2005). Psicología de la emoción: el proceso emocional. Página de Mariano Chóliz Montañés. www. uv.es/=choliz

Clore, G. L. (2005). The influence of affect on attitude. In D. Albarracín, B. T. Johnson, \& M. P. Zanna, The hanbook of attitudes (pp. 437-489). Lawrence Erlbaum Associates, Publishers.

Coates, A., Castro, A., Marmot, M., Mujica, O., Eijkemans, G., \& Victora, C. (2020). Just societies: A new vision for health equity in the Americas after COVID-19. Revista Panamena Salud Pública, 44, 1-4. https://doi.org/10.26633/RPSP.2020.137

Comisión Interamericana de Mujeres. (2020). COVID-19 en la vida de las mujeres: Razones para reconocer los impactos diferenciados. Secretaría General de la Organización de los Estados Americanos (SG/OEA). https://www.oas.org/es/cim/docs/ArgumentarioCOVID19-ES.pdf

Cuiyan, P. R., Wan, X., Tan, Y., Xu, L., Ho, C. S., \& Ho, R. C. (2020). Immediate psychological responses and associated factors during the initial stage of the 2019 coronavirus disease (COVID-19) epidemic among the general population in China. International Journal of Environmental Research and Public Health, 17(5), 1729. https://doi.org/10.3390/ijerph17051729

Damasio, A. (2009). En busca de Spinoza. Neurobiología de la emoción. Crítica.

De Jaegher, H. (2019). Loving and knowing: reflections for an engaged epistemology. Phenomenology and the Cognitive Sciences, 20, 847-870 http://doi.org/10.1007/s11097-019-09634-5

De Sousa, R. (1987). The Rationality of Emotion. MIT Press.

Domínguez, A., Aguilera, S., Acosta, T., Navarro, G., \& Ruiz, Z. (2012). La deseabilidad social revalorada: más que una distorsión, una necesidad de aprobación social. Acta de investigación Psicológica, 2, 808-824.

Emerson, K. (2020). Coping with being cooped up: Social distancing during COVID-19 among 60+ in the United States. Revista Panameña de Salud pública, 44, 1-7.

Fernández González , L., \& Bravo Valenzuela, P. (2020). Expertos y redes sociales: ¿Cómo comunicarnos en tiempos de pandemia? Revista médica de Chile, 148(4), 560-561. http://dx.doi.org/10.4067/s003498872020000400560

Gómez, R. (2012). Gestión de políticas públicas: aspectos operativos. Revista Facultad Nacional Salud Pública, 30(2), 223-236. 
Guenther, L. (2013). Solitary Confinement: Social Death and Its Afterlives. Minnesota University Press

Hawryluck, L., Gold, W. L., Robinson, S., Pogorski, S., Galea, S., \& Styra, R. (2004). SARS control and psychological effects of quarantine, Toronto, Canada. Emerging infectious diseases, 10(7), 1206-1212. https://doi.org/10.3201/eid1007.030703

Jímenez, Á., Duarte, F., \& Rojas, G. (2020, junio 20). Sindemia, la triple crisis social, sanitaria y económica; y su efecto en la salud mental. Ciper académico. https://cutt.ly/WYXATDs

Johnson, R., Onwuegbuzie, A., \& Turner, L. (2007). Toward a definition of mixed Methods research. Journal of Mixed Methods research, 1(2), 112-133. https://doi.org/10.1177\%2F1558689806298224

Johnsonn, M., Saletti-Cuesta, L., \& Tumas, N. (2020). Emociones, preocupaciones y reflexiones frente a la pandemia del COVID-19. Ciência \& Saude Coletiva, 25(1), 2447-2456. http://dx.doi.org/10.1590/141381232020256.1.10472020

Leiva, A. M., Nazar, G., Martinez-Sangüinetti, M. A., Petermannn-Rocha, F., Richezza, J., \& Celis-Morales, C. (2020). Dimensión psicosocial de la pandemia: la otra cara del Covid-19. Ciencia y enfermería, 26(10). http://dx.doi.org/10.29393/ce26-3dpal60003

Mazza, C., Ricci, E., Biondi, S. C., Ferracuti, S., Napoli, C., \& Roma, P. (2020). A Nationwide Survey of Psychological Distress among Italian People during the COVID-19 Pandemic: Immediate Psychological Responses and Associated Factors. Environmental research and public health, 17(9), 3165. https://doi.org/10.3390/ijerph17093165

Nussbaum, M. (2008). Paisajes del pensamiento. La Inteligencia de las emociones. Barcelona: Paidós.

OMS. (2020). Preventing and Managing Covid19 Across Long-Term Care Services. Organización Mundial de la Salud.

Ortega, M. (2020). COVID-19: la nueva enfermedad X. Sanidad Militar, 76(1), 5-7. http://doi.org/10.4321/ s1887-85712020000100001

Ortony, A., \& Clore, G. (2015). Can an Apraisal Model Be Compatible with Psychological Constructonism? En L. Feldman Barrett, \& J. A. Rusell, The psychological constrution of emotion (pp. 305-333). Guilford Press.

Podestá Arzubiaga, J. (2001). Problematización de las políticas públicas desde la óptica regional. 9(15), 163-175. http://dx.doi.org/10.4067/S0718-22362001000200007

Ponce de León, L. (2020). Reflexiones sobre COVID-19 y población vulnerable: ¿Estado de Bienestar o Neoliberalismo? Ehquidad. International Welfare Policies and Social Work Journal, 14, 13-36. https://doi. org/10.15257/ehquidad.2020.0010

Pozo, J. I., Scheuer, N., Pérez Echeverría, M. d., Mateos, M., Martín, E., \& de la Cruz, M. (2006). Nuevas Formas de Pensar la Enseñanza y el Aprendizaje. Las concepciones de Profesores y Alumnos. Barcelona: GRAÓ.

Rousseau, D. M., Sitkin, S. B., Burt, R. S., \& Camerer, C. (1998). Not so different after all: A cross-discipline view of trust. Academy of Management Review, 23(3), 393-404.

Salari, N., Hosseinian-Far, A., Jalali, R., Vaisi-Raygani, Rasoulpoor, S., Mohammadi, M., Rasoulpoor, S., \& Khaledi-Paveh, B. (2020). Prevalence of stress, anxiety, depression among the general population during the COVID-19 pandemic: a systematic review and meta-analysis. Global Health, 57(16). https:// doi.org/10.1186/s12992-020-00589-w 
Sandín, B., \& Chorot, P. (2017). Cuestionario de Sucesos Vitales (CSV). Revista de Psicopatología y Psicología, 22, 95-115. https://doi.org/10.5944/rppc.vol.22.num.2.2017.19729

Sandín, B., Valiente, R., García-Escalera, J., \& Chorot, P. (2020). Impacto psicológico de la pandemia de COVID-19: Efectos negativos y positivos en población española asociados al periodo de confinamiento nacional. Revista de Psicopatología y Psicología, 25(1), 1-22. https://doi.org/10.5944/ rppc. 27569

Santos Padrón, H. (2011). Los determinantes sociales, las desigualdades en salud y las políticas, como temas de investigación. Revista Cubana de Salud Pública, 37(2). http://scielo.sld.cu/scielo. php?script=sci_artt

Solomon, R. (2007). Etica emocional: una teoría de los sentimientos. Paidos.

Taylor, M., Agh, K., Stevens, G., \& Raphael, B. (2008). Factors influencing psychological distress during a disease epidemic: data from Australia's first outbreak of equine influenza. BMC Public Health, 8, 347. https://doi.org/10.1186/1471-2458-8-347

Truco, M. (2002). Estrés y trastornos mentales: aspectos neurobiologicos y psicosociales. Revista chilena de neuropsiquiatría, 40(2), 8-19. http://dx.doi.org/10.4067/S0717-92272002000600002.

United Nations. (2020). Shared responsibility, Global solidarity: Responding to the socio-economic impacts of COVID-19. ONU. https://cutt.ly/qYXDal8

Verdugo, L. M. (2021). Habitabilidad de la vivienda en tiempos de pandemia por Covid-19 en México. El caso de Culiacán. EHQUIDAD. Revista Internacional De Políticas De Bienestar Y Trabajo Social, 15, 77-112. https://doi.org/10.15257/ehquidad.2021.0004

World Health Organization. (2020). Pandemic fatigue - reinvigorating the public to prevent Covid-19. WHO Regional Office for Europe.

\section{AUTORES}

Rodolfo Bachler Silva. Doctor en Psicología Educativa, Magíster en Estudios Cognitivos, Psicólogo. Académico e investigadora de la Escuela de Psicología de la Universidad Mayor.

Pablo Segovia-Lagos. Doctor en Sociología, Magíster en Metodologías de Investigación, Psicólogo. Académico e investigadora de la Escuela de Psicología de la Universidad Mayor. Coordinadora de la Unidad de Investigación Escuela de Psicología.

Constanza Carter. Periodista e investigadora independiente. 